\title{
Association between serum bilirubin and albuminuria in type 2 diabetes mellitus and diabetic nephropathy
}

\author{
Suryapriya Rajendran ${ }^{1}$, Manju M.,", , Sasmita Mishra ${ }^{3}$, Rakesh Kumar ${ }^{4}$ \\ ${ }^{\mathbf{1}, \mathbf{4}}$ Assistant Professor, ${ }^{2}$ Associate Professor, ${ }^{3}$ Professor and HOD, Dept. of Biochemistry, ${ }^{4}$ Dept. of General Medicine, Aarupadai \\ Veedu Medical College and Hospital, Puducherry, India.
}

*Corresponding Author:

Email: drmanjumay1@gmail.com

Received: $16^{\text {th }}$ February, 2018

Accepted: $20^{\text {th }}$ March, 2018

\begin{abstract}
Introduction: Diabetic nephropathy develops due to oxidative stress and inflammation resulting from chronic hyperglycemia. Bilirubin, a product of heme catabolism is found to have antioxidant and anti-inflammatory properties. Though previous studies have examined the relationship between total bilirubin and diabetic nephropathy, very few studies have focused on indirect and direct bilirubin levels. Hence, the present study aimed to compare serum bilirubin (total, indirect and direct) levels between nondiabetics, type 2 diabetics and diabetic nephropathy subjects and also to correlate albuminuria with serum bilirubin in type 2 diabetics and diabetic nephropathy subjects.

Materials and Methods: 50 non-diabetics, 50 type 2 diabetics and 50 diabetic nephropathy subjects were included in the study. Fasting blood glucose, $\mathrm{HbA}_{1 \mathrm{C}}$, serum bilirubin (total, indirect and direct), serum creatinine, urine microalbumin and urine creatinine were measured. Estimated glomerular filtration rate (eGFR) and urine albumin creatinine ratio (ACR) was calculated.

Results: Total bilirubin, direct and indirect bilirubin were significantly decreased in type 2 diabetics and diabetic nephropathy subjects compared to non-diabetics. Total bilirubin and indirect bilirubin were also significantly decreased in diabetic nephropathy subjects compared to type 2 diabetics. Total bilirubin, direct and indirect bilirubin showed significant negative correlation with albuminuria (Urine ACR) in type 2 diabetics and diabetic nephropathy subjects.

Conclusion: Our study suggests that low bilirubin levels might be a risk factor for the development of type 2 diabetes and diabetic nephropathy. Bilirubin can be used as a marker to predict the risk of developing type 2 diabetes among general population and diabetic nephropathy among type 2 diabetes patients.
\end{abstract}

Keywords: Albuminuria, Antioxidant, Bilirubin, Diabetes mellitus, Diabetic nephropathy.

\section{Introduction}

Diabetic nephropathy is one of the major complications of type 2 diabetes mellitus. Nephropathy occurs in about $20-40 \%$ of patients with type 2 diabetes mellitus and it is the leading cause of end stage renal disease around the world. ${ }^{1-2}$ Diabetic nephropathy develops due to oxidative stress and inflammation resulting from chronic hyperglycemia. ${ }^{2-3}$ Diabetic nephropathy is diagnosed by elevated urinary albumin creatinine ratio (albuminuria) and/or reduced estimated glomerular filtration rate. ${ }^{4}$ The only option available to prevent this complication is strict glycemic control. But nephropathy is also seen to occur in patients under strict glycemic control. ${ }^{3}$ So there is a need to identify a novel biomarker that can predict the risk for diabetic nephropathy.

Bilirubin is a product of heme catabolism by heme oxygenase and it is excreted by liver cells. ${ }^{5}$ Previously, bilirubin is thought to be a toxic waste product, ${ }^{6}$ but recent studies have reported that bilirubin has antioxidant, anti-inflammatory and antiapoptotic properties. ${ }^{2}$ Both direct and indirect bilirubin are found to have these properties. ${ }^{7}$ Previous studies have found that serum total bilirubin levels are inversely associated with the risk of diabetic nephropathy. Higher total bilirubin levels were found to be protective against diabetic nephropathy. ${ }^{2}$

Though there are studies that focus on total bilirubin levels, very few studies have focused on indirect and direct bilirubin levels ${ }^{2}$ and the studies are also lacking in Indian population. Hence, the present study aimed to compare serum bilirubin (total, indirect and direct) levels between non-diabetics, type 2 diabetics and diabetic nephropathy subjects and also to study the correlation of albuminuria with serum bilirubin levels in type 2 diabetics and diabetic nephropathy subjects.

\section{Materials and Methods}

The study was a hospital based cross-sectional study conducted in the Department of Biochemistry, Aarupadai Veedu Medical College \& Hospital, Puducherry, India. The study was approved by our Institutional Research Committee and Institute Ethics Committee (human studies). The study has been performed in accordance with the ethical standards laid down in the 1964 Declaration of Helsinki and its later amendments.

One hundred and fifty subjects aged 30 years and above were recruited from the department of Medicine \& Diabetology (both outpatients and inpatients) at 
Aarupadai Veedu Medical College \& Hospital, Puducherry. The study subjects were divided into three groups: non-diabetics (age and sex matched fifty healthy subjects), type 2 diabetics $(\mathrm{n}=50)$ and diabetic nephropathy $(\mathrm{n}=50)$. Patients with liver diseases, hemolytic diseases, hypertension, cardiovascular disease and nondiabetic chronic kidney disease were excluded from the study.

After obtaining written informed consent from the study subjects, a detailed clinical history was collected from them through a pre-tested, semi-structured questionnaire. Height and weight were measured from all the study subjects and BMI was calculated by the formula: weight in $\mathrm{kg}$ / height in $\mathrm{m}^{2}$. Blood pressure was measured twice in right arm in sitting position using standard mercury sphygmomanometer (after $10 \mathrm{~min}$ rest).

$5 \mathrm{~mL}$ of fasting venous blood sample and urine samples were collected from all the study subjects. Fasting blood glucose, $\mathrm{HbA}_{1 \mathrm{C}}$, serum bilirubin (total, indirect and direct), serum creatinine, urine microalbumin and urine creatinine were assayed in the fully automated biochemistry analyzer (Mindray BS380). eGFR was calculated using MDRD equation $\mathrm{eGFR}=186.3 \times \quad(\text { plasma creatinine })^{-1.154} \times$ age $^{-0.203}$ $(\times 0.742$ for women). Urine albumin creatinine ratio (ACR) was calculated using the values of urine microalbumin and urine creatinine.

\section{Statistical analysis}

Normally distributed variables are expressed as mean with standard deviation and non-normally distributed variables are expressed as median (interquartile range). Comparison of anthropometric variables and biochemical parameters among nondiabetics, type 2 diabetics and diabetic nephropathy subjects were assessed by one-way ANOVA (for normal data) and Kruskal-Wallis test (for non-normal data). Correlation analysis of albuminuria (Urine ACR) with total, direct and indirect bilirubin was done using Spearman Rank correlation. A p value of $<0.05$ was considered as statistically significant. All the statistical analysis were performed by using SPSS software version 16 (SPSS Inc., USA).

\section{Results}

A total of 150 subjects were included in the study. Characteristics of study population are shown in Table 1. No significant difference was found in age, height, weight, body mass index, systolic and diastolic blood pressure among non-diabetics, type 2 diabetics and diabetic nephropathy subjects.

Fasting blood glucose and $\mathrm{HbA}_{1 \mathrm{c}}$ were significantly increased in type 2 diabetics and diabetic nephropathy subjects compared to non-diabetics. Diabetic nephropathy subjects had significantly increased urine ACR levels compared to non-diabetics and type 2 diabetics. Total bilirubin, direct and indirect bilirubin were significantly decreased in type 2 diabetics and diabetic nephropathy subjects compared to nondiabetics. Total bilirubin and indirect bilirubin were also significantly decreased in diabetic nephropathy subjects compared to type 2 diabetics (Table 2). Comparison of total, direct and indirect bilirubin levels between non-diabetics, type 2 diabetics and diabetic nephropathy subjects is shown in Fig. 1.

Total bilirubin, direct and indirect bilirubin showed significant negative correlation with albuminuria (urine ACR) in type 2 diabetics and diabetic nephropathy subjects. Correlation analysis between bilirubin and albuminuria is shown in Table 3, Fig. 2 and Fig. 3.

Table 1: Characteristics of study population

Data are expressed as mean \pm SD

\begin{tabular}{|l|c|c|c|}
\hline \multicolumn{1}{|c|}{ Variables } & $\begin{array}{c}\text { Non diabetics } \\
(\mathbf{n = 5 0})\end{array}$ & $\begin{array}{c}\text { Type 2 diabetics } \\
(\mathbf{n = 5 0})\end{array}$ & $\begin{array}{c}\text { Diabetic } \\
\text { nephropathy } \\
(\mathbf{n = 5 0})\end{array}$ \\
\hline Age (years) & $50.9 \pm 8.8$ & $53.7 \pm 14.6$ & $56.7 \pm 14.3$ \\
\hline Height $(\mathrm{cm})$ & $162.5 \pm 7.9$ & $162.3 \pm 7.7$ & $163.2 \pm 7.6$ \\
\hline Weight $(\mathrm{kg})$ & $67.3 \pm 10.8$ & $68.8 \pm 8.9$ & $66.6 \pm 9.4$ \\
\hline Body mass index $\left(\mathrm{kg} / \mathrm{m}^{2}\right)$ & $25.4 \pm 3.2$ & $26.1 \pm 2.5$ & $25 \pm 3.2$ \\
\hline Systolic blood pressure $(\mathrm{mm} \mathrm{Hg})$ & $117 \pm 8.1$ & $117.5 \pm 10.2$ & $116.4 \pm 6.9$ \\
\hline Diastolic blood pressure $(\mathrm{mm} \mathrm{Hg})$ & $75 \pm 6.7$ & $75.8 \pm 6.0$ & $74.4 \pm 6.1$ \\
\hline
\end{tabular}


Table 2: Comparison of biochemical parameters between non-diabetics, type 2 diabetics and diabetic nephropathy subjects

\begin{tabular}{|l|c|c|c|}
\hline \multicolumn{1}{|c|}{ Variables } & $\begin{array}{c}\text { Non diabetics } \\
(\mathbf{n = 5 0})\end{array}$ & $\begin{array}{c}\text { Type 2 diabetics } \\
(\mathbf{n = 5 0})\end{array}$ & $\begin{array}{c}\text { Diabetic nephropathy } \\
(\mathbf{n = 5 0})\end{array}$ \\
\hline Fasting blood glucose $(\mathrm{mg} / \mathrm{dL})$ & $77.4 \pm 9.3$ & $158.8 \pm 67.5^{*}$ & $168.6 \pm 81.2^{*}$ \\
\hline $\mathrm{HbA}_{1 \mathrm{C}}(\%)$ & $5.3 \pm 0.8$ & $7.6 \pm 1.4^{*}$ & $7.9 \pm 1.6^{*}$ \\
\hline Creatinine $(\mathrm{mg} / \mathrm{dL})$ & $0.72 \pm 0.13$ & $0.81 \pm 0.21$ & $0.89 \pm 0.58$ \\
\hline eGFR $\left(\mathrm{mL} / \mathrm{min} / 1.73 \mathrm{~m}^{2}\right)$ & $115.9 \pm 30.3$ & $105.4 \pm 41.6$ & $103.5 \pm 42.3$ \\
\hline Urine ACR $(\mathrm{mg} / \mathrm{g}$ of cr) & $20.5(10)$ & $23.5(10)$ & $68(81)^{*} \#$ \\
\hline Total Bilirubin $(\mathrm{mg} / \mathrm{dL})$ & $0.89 \pm 0.13$ & $0.74 \pm 0.29^{*}$ & $0.61 \pm 0.29^{* \#}$ \\
\hline Direct Bilirubin $(\mathrm{mg} / \mathrm{dL})$ & $0.27 \pm 0.06$ & $0.21 \pm 0.08^{*}$ & $0.19 \pm 0.07^{*}$ \\
\hline Indirect Bilirubin $(\mathrm{mg} / \mathrm{dL})$ & $0.62 \pm 0.12$ & $0.53 \pm 0.24^{*}$ & $0.42 \pm 0.25^{* \#}$ \\
\hline
\end{tabular}

eGFR - estimated glomerular filtration rate; ACR - albumin creatinine ratio; Data are expressed as mean \pm SD; Urine ACR expressed as median (interquartile range); $* \mathrm{p}<0.05$ compared to non diabetics; $\# \mathrm{p}<0.05$ compared to type 2 diabetics

Table 3: Correlation of bilirubin with albuminuria (urine ACR) in type 2 diabetics and diabetic nephropathy subjects

\begin{tabular}{|l|c|c|c|c|}
\hline \multirow{2}{*}{\multicolumn{1}{|c|}{ Variables }} & \multicolumn{2}{|c|}{ Type 2 diabetics } & \multicolumn{2}{c|}{ Diabetic nephropathy } \\
\cline { 2 - 5 } & r value & p value & r value & p value \\
\hline Total Bilirubin & -0.654 & $<0.001^{*}$ & -0.509 & $<0.001^{*}$ \\
\hline Direct Bilirubin & -0.498 & $0.002^{*}$ & -0.353 & $0.012^{*}$ \\
\hline Indirect Bilirubin & -0.603 & $<0.001^{*}$ & -0.558 & $<0.001^{*}$ \\
\hline
\end{tabular}

$* \mathrm{p}$ value $<0.05$, statistically significant

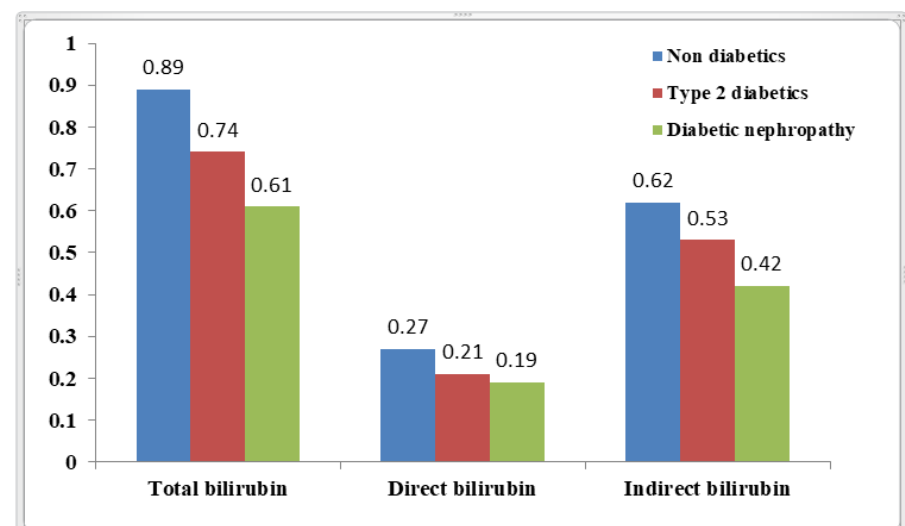

Fig.1: Comparison of total, direct and indirect bilirubin between non diabetics, type 2 diabetics and diabetic nephropathy subjects

(A)

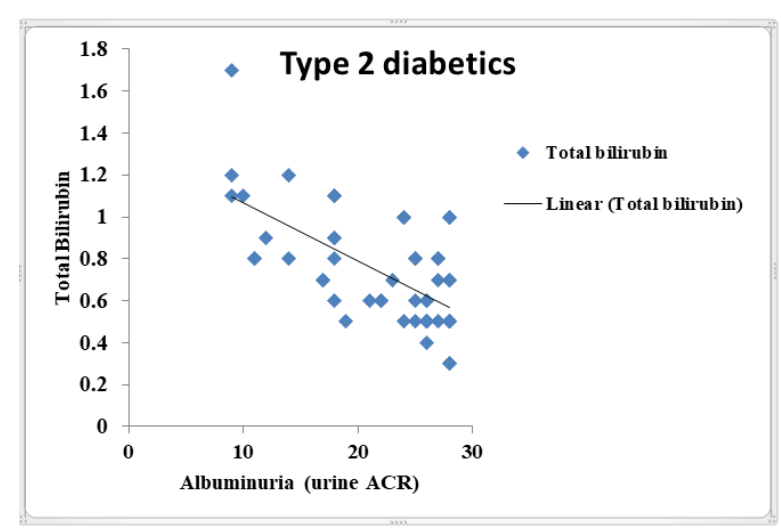


(B)

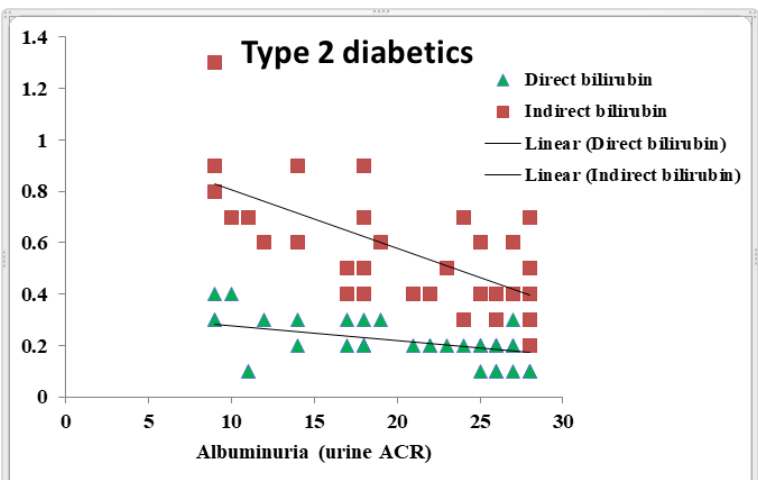

Fig. 2: (A) Correlation between total bilirubin and albuminuria (urine ACR) in type 2 diabetics, (B) Correlation between direct and indirect bilirubin and albuminuria (Urine ACR) in type 2 diabetics

(A)

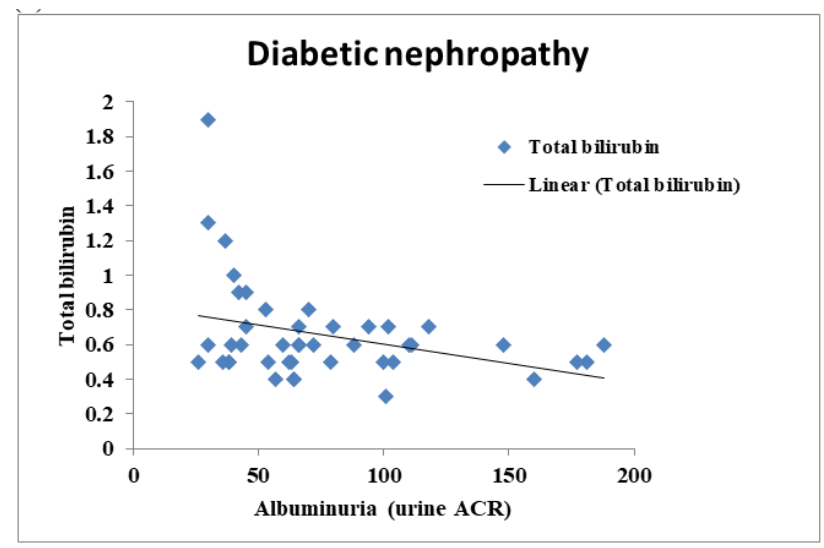

(B)

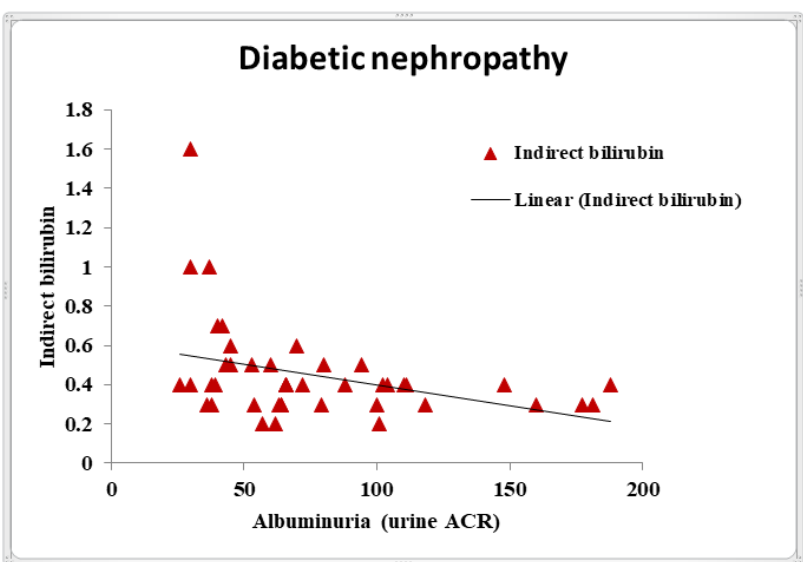

Fig. 3: (A) Correlation between total bilirubin and albuminuria (urine ACR) in diabetic nephropathy subjects, (B) Correlation between indirect bilirubin and albuminuria (Urine ACR) in diabetic nephropathy subjects

\section{Discussion}

This study was done to compare serum bilirubin (total, indirect and direct) levels between non-diabetics, type 2 diabetics and diabetic nephropathy subjects and also to correlate albuminuria (urine ACR) with serum bilirubin levels in type 2 diabetics and diabetic nephropathy subjects.

In this study, we found that total bilirubin and its sub types, both direct and indirect bilirubin were significantly decreased in type 2 diabetics, compared to non-diabetics. This suggests that higher bilirubin might prevent the development of type 2 diabetes. Similar 
findings were observed in a study conducted in Korean men, where they found a negative association between elevated serum bilirubin level and development of type 2 diabetes. ${ }^{8}$ Inverse correlation was also seen between bilirubin and the prevalence of type 2 diabetes mellitus in Korean men and women. ${ }^{6}$

A state of chronic hyperglycemia, seen in type 2 diabetes leads to the increased production of free radicals from mitochondria. This occurs most commonly in vascular endothelial cells, resulting in vascular dysfunction, a characteristic feature seen in type 2 diabetes as well as in diabetic complications. ${ }^{5}$ Bilirubin, being a potent anti-oxidant, might compensate this oxidative stress and prevent the development of type 2 diabetes. ${ }^{9}$

On comparing bilirubin levels between type 2 diabetics and diabetic nephropathy, we found that total bilirubin and indirect bilirubin, but not direct bilirubin, were significantly decreased in diabetic nephropathy subjects compared to type 2 diabetics. Though, total bilirubin includes the sum of direct and indirect bilirubin, indirect bilirubin accounts for $96 \%$ of total bilirubin. So, inspite of the fact that both direct and indirect bilirubin have beneficial properties, ${ }^{7}$ in our study, only indirect bilirubin was significantly decreased in diabetic nephropathy subjects. This may be due to higher proportion of indirect bilirubin in serum. ${ }^{7}$ We also found a significant negative correlation between albuminuria and total, direct and indirect bilirubin in type 2 diabetics as well as in diabetic nephropathy subjects.

Fukui et al, ${ }^{10}$ in his study conducted among 633 type 2 diabetes patients in Japan, also found lower bilirubin levels in patients with diabetic nephropathy than patients without diabetic nephropathy, and also negative association of bilirubin with albuminuria, similar to our findings. Another post hoc analysis also showed that bilirubin is inversely associated with diabetic nephropathy progression. ${ }^{1}$ Toya et al, ${ }^{11}$ in his study found that elevated bilirubin levels were associated with decreased risk of progression from microalbuminuria to macroalbuminuria. Okada et al, ${ }^{12}$ reported that decreased bilirubin levels might be a risk factor for the development of albuminuria in type 2 diabetes patients.

These previous studies have studied only the relationship between total bilirubin and diabetic nephropathy, but hardly two or three studies have investigated the relationship between indirect and direct bilirubin and diabetic nephropathy. Wang et $\mathrm{al}^{2}{ }^{2}$ in his study showed that elevated total and indirect bilirubin levels significantly decreased the risk of diabetic nephropathy development in type 2 diabetes patients. Other studies conducted in type 1 diabetes patients, also showed negative correlation of total and indirect bilirubin levels with albuminuria., ${ }^{4,13}$

Several mechanisms have been proposed to explain the protective role of bilirubin against the development of albuminuria and diabetic nephropathy. ${ }^{7}$ Oxidative stress is one of the major mechanisms for the development and progression of diabetic nephropathy. ${ }^{1}$ Bilirubin is found to be an endogenous anti-oxidant, ${ }^{1,14-}$ 15 and a study conducted in rodents, suggested that bilirubin protects against the development of diabetic nephropathy by inhibiting NADPH oxidase. ${ }^{1}$ Kumar et al, ${ }^{16}$ showed negative correlation of bilirubin with oxidative stress marker, malondialdehyde and positive correlation of bilirubin with anti-oxidant enzyme activities, supporing the anti-oxidant role of bilirubin.

Bilirubin also has anti-inflammatory property by disrupting the trafficking of leucocytes to the renal interstitium, by inhibiting vascular cell adhesion protein mediated signalling. Bilirubin does so, by inhibiting the gene up regulation of endothelial adhesion molecule like E-selectin, which plays an important role in infiltrating leucocytes into renal interstitium. ${ }^{7}$

In addition to anti-oxidant and anti-inflammatory properties, bilirubin also has anti complement property and cytoprotective effect by inhibiting protein kinase C. ${ }^{10,17}$ Hence, bilirubin might prevent the development of type 2 diabetes and diabetic nephropathy by inhibiting oxidative stress and inflammation.

\section{Conclusion}

Our study suggests that low bilirubin levels might be a risk factor for the development of type 2 diabetes and diabetic nephropathy. Bilirubin being an inexpensive, easily measurable and routinely performed parameter in the laboratory can be used as a marker to predict the risk of developing type 2 diabetes among general population and also to predict the risk of developing diabetic nephropathy among type 2 diabetes patients. Further studies are required to determine whether bilirubin levels can be a therapeutic target for preventing the development of type 2 diabetes and diabetic nephropathy.

\section{References}

1. Riphagen IJ, Deetman PE, Bakker SJ, Navis G, Cooper ME, Lewis JB, de Zeeuw D, Lambers Heerspink HJ. Bilirubin and progression of nephropathy in type 2 diabetes: a post hoc analysis of RENAAL with independent replication in IDNT. Diabetes. 2014;63:2845-53.

2. Wang J, Li Y, Han X, Hu H, Wang F, Yu C, Li X, Yang K, Yuan J, Yao P, Miao X, Wei S, Wang Y, Chen W, Liang Y, Zhang X, Guo H, Pan A, Yang H, Wu T, He M. Association between serum bilirubin levels and decline in estimated glomerular filtration rate among patients with type 2 diabetes. J Diabetes Complications. 2016;30:1255-60.

3. Kim ES, Lee SW, Mo EY, Moon SD, Han JH. Inverse association between serum total bilirubin levels and diabetic peripheral neuropathy in patients with type 2 diabetes. Endocrine. 2015;50:405-12.

4. Nishimura T, Tanaka M, Sekioka R, Itoh H. Serum bilirubin concentration is associated 
with eGFR and urinary albumin excretion in patients with type 1 diabetes mellitus. J Diabetes Complications. 2015;29(8):1223-7.

5. Ahn KH, Kim SS, Kim WJ, Kim JH, Nam YJ, Park SB, Jeon YK, Kim BH, Kim IJ, Kim YK.

Low serum bilirubin level predicts the development of chronic kidney disease in patients with type 2 diabetes mellitus. Korean J Intern Med. 2017;32(5):875-82.

6. Han SS, Na KY, Chae DW, Kim YS, Kim S, Chin HJ. High serum bilirubin is associated with the reduced risk of diabetes mellitus and diabetic nephropathy. Tohoku J Exp Med 2010;221:133-40.

7. Wang J, Guo P, Gao Z, Zhou B, Ren L, Chen Y, Zhou Q. Elevated bilirubin levels and risk of developing chronic kidney disease: a dose-response meta-analysis and systematic review of cohort studies. Int Urol Nephrol. 2018;50(2):275-87.

8. Jung CH, Lee MJ, Kang YM, Hwang JY, Jang JE, Leem $\mathrm{J}$, et al. Higher serum bilirubin level as a protective factor for the development of diabetes in healthy Korean men: a 4 year retrospective longitudinal study. Metabolism. 2014;63:87-93.

9. Abbasi A, Deetman PE, Corpeleijn E, Gansevoort RT, Gans RO, Hillege HL, van der Harst P, Stolk RP, Navis G, Alizadeh BZ, Bakker SJ. Bilirubin as a potential causal factor in type 2 diabetes risk : a Mendelian randomization study. Diabetes. 2015;64(4):1459-69.

10. Fukui M, Tanaka M, Shiraishi E, Harusato I, Hosoda H, Asano M, et al. Relationship between serum bilirubin and albuminuria in patients with type 2 diabetes. Kidney Int. 2008;74:1197-201.

11. Toya K, Babazono T, Hanai K, Uchigata Y. Association of serum bilirubin levels with development and progression of albuminuria, and decline in estimated glomerular filtration rate in patients with type 2 diabetes mellitus. $J$ Diabetes Investig. 2014;5:228-35.

12. Okada H, Fukui M, Tanaka M, et al. Low serum bilirubin concentration is a novel risk factor for the development of albuminuria in patients with type 2 diabetes. Metabolism. 2014;63(3):409-14.

13. Li X, Zhang L, Chen H, Guo K, Yu H, Zhou J, Li M, Li Q, Li L, Yin J, Liu F, Bao Y, Han J, Jia W. Relationship between serum bilirubin concentrations and diabetic nephropathy in Shanghai Han's patients with type 1 diabetes mellitus. BMC Nephrol. 2017;18(1):114.

14. Mashitani T, Hayashino Y, Okamura S, Tsujii S, Ishii H. Correlations between serum bilirubin levels and diabetic nephropathy progression among Japanese type 2 diabetic patients: a prospective cohort study (Diabetes Distress and Care Registry at Tenri [DDCRT 5]). Diabetes Care 2014;37(1):252-8.

15. Katoh T, Kawamoto R, Kohara K, Miki T. Association between serum bilirubin and estimated glomerular filtration rate among diabetic patients. Int Sch Res Notices. 2015;26:480-18.

16. Kumar A, Pant P, Basu S, Rao GR, Khanna HD. Oxidative stress in neonatal hyperbilirubinemia. J Trop Pediatr. 2007;53:69271.

17. Tanaka M, Fukui M, Okada H, Senmaru T, Asano M, Akabame S, Yamazaki M, Tomiyasu K, Oda Y, Hasegawa G, Toda H, Nakamura N. Low serum bilirubin concentration is a predictor of chronic kidney disease. Atherosclerosis. 2014;234(2):421-5. 